\title{
Resolution of Obstructive Atelectasis with High Flow Nasal Cannula
}

\author{
Javier Pozas, Diurbis Velasco, Salvador Díaz-Lobato* \\ Pneumological Department, Ramón y Cajal Univeritary Hospital, Madrid, Spain \\ *Corresponding Author: Salvador Díaz-Lobato, Pneumology Department, Ramón y Cajal Universitary \\ Hospital, Madrid, Spain, Email: sdiazlobato@gmail.com
}

\begin{abstract}
High Flow Nasal Cannula (HFNC) oxygen therapy is a technique commonly used in daily clinical practice in several hospital areas. We present here the case of a 45-year-old patient with a history of severe restrictive lung disease due to a congenital spinal muscular atrophy, who developed acute respiratory failure due to complete atelectasis of the left lung associated with community-acquired pneumonia. The patient presented significant work-of-breathing, severe desaturation and hypercapnic acidosis. The chest X-ray showed a complete atelectasis of the left lung. After being stabilized at the ICU, the patient was transferred to the pneumologyward where HFNC was initiated. Progress was favorable, with clear clinical and radiological improvement. The chest X-ray performed four days later showed an important improvement of the atelectasis. These data suggest that HFNC may be useful in the treatment of atelectasis in some critical patients.
\end{abstract}

\section{INTRODUCTION}

High Flow Nasal Cannula (HFNC) oxygen therapy is a recent technique that delivers a high flow of heated and humidified gas to the patient [1]. Compared to noninvasive ventilation (NIV), HFNC has been proved to be an effective alternative treatment for acute respiratory failure. HFNC also has a significant number of physiological advantages compared with other commonly used oxygen-based therapies, including PEEP, reduced anatomical dead space, constant $\mathrm{FiO}_{2}$ and it also is better tolerated than NIV [2]. After a thorough bibliographical research, an association between the clinical use of HFNC and the improvement of lung atelectasis has not been found. We present the case of a 45 year old neuromuscular patient with obstructive atelectasis of the left lung and respiratory acidosis, resolved with the use of HNFC.

\section{CASE REPORT}

A 45-year old man who suffers from congenital spinal muscular atrophy. He is followed up by the Pneumology Department of our Hospital for a severe restrictive lung disease secondary to his neuromuscular condition. However, due to an adequate clinical and gasometry stability, the use of home NIV was not indicated. On April 2018, the patient presented to the hospital referring dyspnea and clinical signs that suggested the diagnosis of pneumonia. He was admitted in the pneumologyward but due to a worsening of the gasometric parameters as well as a deterioration of the clinical status, he was admitted to the ICU of our facility. At his arrival, temperature was $38,1^{\circ} \mathrm{C}$. The patient presented as well hypotension (blood pressure was $90 / 50 \mathrm{mmHg}$ ), tachycardia (120 beats per minute), tachypnea (28 breaths per minute) and the pulsioximetry oxygen saturation $(\mathrm{SpO} 2)$ was $81 \%$ despite administration of noninvasive ventilation. When it came to physical examination, the patient had diminished normal breath sounds with abundant abnormal sounds.

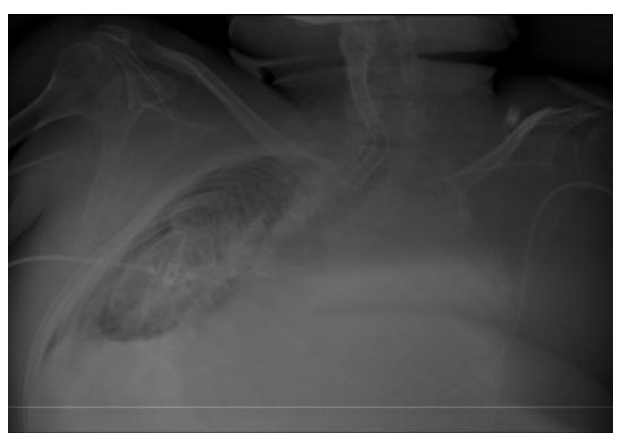

Figure1. Complete atelectasis of the left lung and partial atelectasis of the right lung

During the patient's transfer to ICU, an accidental fall occurred leading to a subtrochanteric fracture that was surgically treated. After 7 days of orotracheal intubation, a tracheotomy was performed and mechanical 
ventilation was started, showing $\mathrm{pH}$ : 7.36; $\mathrm{PaCO}_{2}: 55 \mathrm{mmHg} ; \quad \mathrm{PaO}_{2}: 42 \mathrm{mmHg} ; \mathrm{HCO}_{3}{ }^{-}$: $31.1 \mathrm{mmol} / \mathrm{l}$ at blood gases. A nasogastric tube was inserted. Despite the efforts, decannulation was unsuccessful, hence it was decided to transfer the patient to the pneumology ward after 40 days at the ICU. At his arrival, chest Xray showed a complete atelectasis of the left lung [Fig. 1]

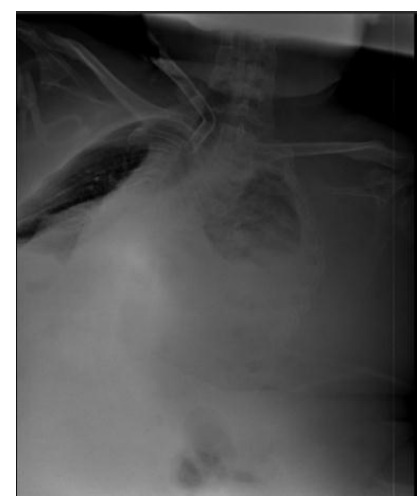

Figure2. Significant improvement of both lungs after 4 days of HFNC therapy

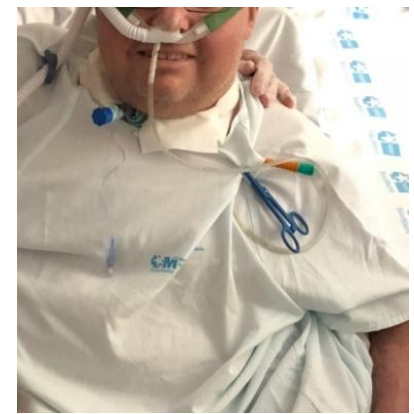

Figure3. Patient receiving high flow nasal cannula therapy with the tracheostoma occluded with a plug

In the ward, the cuff of the tracheostomy tube was deflated, the tracheostoma was occluded with a plug, and invasive ventilation through the tracheostomy tube was substituted by HFNC (setting at 60 liters, $\mathrm{FiO}_{2} 40 \%$ ], allowing the patient to speak again and maintaining good oxygen saturation ( $\mathrm{SpO} 295 \%)$. To improve the expectoration of secretions, a Cough Assist device was used through the tracheotomy cannula, resulting in an increased patient's comfort. A control chest X-Ray was performed four days after the patient's admission into the pneumology ward, showing a significant improvement in the atelectasis of the left lung [Fig. 2]. The patient was finally decannulated, remaining on HFNC till the hospital discharge [Fig. 3].

\section{DISCUSSION}

Atelectasis is a common complication in patients with respiratory diseases, specially in patients tracheostomised and mechanical ventilation. Several techniques have been used in order to prevent it, essentially mechanical insufflation-exsufflation devices such as Cough Assist or respiratory physiotherapy [3]. Since this approach is often overlooked, invasive procedures such as bronchoscopy are commonly performed in these patients.

In our patient, a combination of HFNC and Cough Assist was used in order to improve oxygenation and secretion clearing, resulting in a significant improvement of the atelectasis. HFNC was a good alternative to mechanical ventilation once the patient was stable. No references to the inclusion of HFNC in the treatment of patients with atelectasis were found. Our experience suggest that some specific patients may benefit from HFNC instead of undergoing less innocuous procedures such as bronchoscopy. Further studies are necessary for a better understanding of the role of HFNC in patients with pulmonary atelectasis.

\section{REFERENCES}

[1] Frat JP, Coudroy R, Marjanovic N, Thille AW. High-flow nasal oxygen therapy and noninvasive ventilation in the management of acute hypoxemic respiratory failure. Ann Transl Med. 2017 Jul; 5(14):297.

[2] Nishimura M. High-Flow Nasal Cannula Oxygen Therapy in Adults: Physiological Benefits, Indication, Clinical Benefits, and Adverse Effects. Respiratory Care April 2016, 61 (4) 529-541.

[3] Farrero E, et al. Guidelines for the management of respiratory complications in patients with neuromuscular disease. Spanish Respiratory Society (SEPAR). Arch Bronconeumol. 2013 Jul; 49(7):306-13.

Citation: Javier Pozas, Diurbis Velasco, Salvador Díaz-Lobato. Resolution of Obstructive Atelectasis with High Flow Nasal Cannula. ARC Journal of Clinical Case Reports. 2018; 4(2): 7-8. doi:dx.doi.org/ 10.20431/2455-9806.0402002.

Copyright: (C) 2018 Authors. This is an open-access article distributed under the terms of the Creative Commons Attribution License, which permits unrestricted use, distribution, and reproduction in any medium, provided the original author and source are credited. 